\title{
MINIMAL IMMERSIONS OF CLOSED RIEMANN SURFACES
}

\author{
BY
}

\author{
J. SACKS ${ }^{1}$ AND K. UHLENBECK ${ }^{2}$
}

\begin{abstract}
Let $M$ be a closed orientable surface of genus larger than zero and $N$ a compact Riemannian manifold. If $u: M \rightarrow N$ is a continuous map, such that the map induced by it between the fundamental groups of $M$ and $N$ contains no nontrivial element represented by a simple closed curve in its kernel, then there exists a conformal branched minimal immersion $s: M \rightarrow N$ having least area among all branched immersions with the same action on $\pi_{1}(M)$ as $u$. Uniqueness within the homotopy class of $u$ fails in general: It is shown that for certain 3-manifolds which fiber over the circle there are at least two geometrically distinct conformal branched minimal immersions within the homotopy class of any inclusion map of the fiber. There is also a topological discussion of those 3-manifolds for which uniqueness fails.
\end{abstract}

Introduction. In a previous paper we obtained results on minimal immersions of the two-sphere into compact Riemannian manifolds [18]. Here we extend some of these results to surfaces of higher topological type. The main idea is to reduce the minimal area problem for such surfaces to a variational problem on a moduli space for conformal structures on the surface. The main technical difficulty to overcome is the fact that the moduli spaces are not compact. We overcome this by using a standard compactification of the Riemann moduli space (see Bers [5, 6] and Abikoff [2]) and controlling the behaviour of our variational problem near the boundary points.

Our main result, Theorem 4.4, proves the existence of a conformal branched minimal immersion of a surface $M$ with genus larger than zero corresponding to every homotopy class of maps $u: M \rightarrow N$ which induces an injection $u_{*}: \pi_{1}(M) \rightarrow$ $\pi_{1}(N)$. Here $N$ is any compact Riemannian manifold of dimension larger than two. If $\pi_{2}(N)=0$, then the minimal immersion is actually homotopic to $u$. It was conjectured that in the case where $N$ is hyperbolic, any such minimal immersion would have to be unique within its homotopy class. Surprisingly, this is not necessarily true, as we prove in Corollary 5.5, in which we show that for certain hyperbolic 3-manifolds $N$ and $u: M \rightarrow N$, there are at least two geometrically distinct minimal immersions homotopic to $u$. This nonuniqueness result is obtained by developing a Ljusternik-Schnirelman theory for minimal immersions of surfaces of higher topological type.

Received by the editors February 26, 1981.

1980 Mathematics Subject Classification. Primary 53A10, 58E05: Secondary 57N10.

'Research supported by NSF grants MCS 76-06319 and MCS-8002959.

${ }^{2}$ Research supported by NSF grant MCS 76-07541. 
In $\S 1$ we discuss the existence theory and properties of harmonic maps and recall results from our earlier paper [18]. In $\$ 2$ we state some convergence properties of sequences of harmonic maps proved in [18] and extend earlier results to the case of variable conformal structures on the domain surface in Theorem 2.3. In $\S 3$ we prove a version of our main result for the special case of genus one, where the argument is technically somewhat different, but the result, Theorem 3.3, is essentially the same as the main Theorem 4.4, which is proved in $\$ 4$. A synopsis of definitions and theorems needed from Teichmüller space theory is presented at the beginning of $\S 4$. The earlier mentioned nonuniqueness result, Corollary 5.5 , is proved in $\S 5$ after the required Ljusternik-Schnirelman theory is developed. In $\$ 6$ we give a topological discussion of the nonuniqueness result for a class of compact 3-manifolds. This chapter is in the spirit of topological 3-manifold theory and links up with the work of W. Thurston on 3-manifolds (see [20, 19]).

Theorem 4.4 was also obtained by R. Schoen and S. T. Yau in [17] using somewhat different techniques. In our proof, a minimizing sequence for the variational functional on Teichmüller space is allowed to have a limit on the boundary in order to obtain a contradiction, thus proving the theorem. They used this result to prove that 3-manifolds whose fundamental groups contain surface groups cannot carry a metric of positive scalar curvature.

We would very much like to thank W. Abikoff for teaching us Teichmüller theory and for patiently answering questions and asking fruitful ones of his own. It is also a pleasure to acknowledge very helpful discussions with R. Schoen and S. T. Yau and W. Thurston's assistance in investigating minimal immersions in hyperbolic 3-manifolds.

1. Properties of harmonic maps. Throughout this paper, $M$ will denote a compact orientable surface with a conformal structure $\mu$, and $N$ a compact $C^{\infty}$ Riemannian manifold of dimension at least 3 . In this section we outline the results on harmonic maps that we shall be using in the rest of the paper. A more detailed account and proofs are contained in [18].

Let $s: M \rightarrow N, s \in C^{\infty}(M, N)$ and consider $d s(x) \in T_{x}^{*}(M) \otimes T_{s(x)}(N)$. Using the inner product given by the Riemannian metric on $N$ and the conformal structure $\mu$ on $M$, we may define $I(s)(x)=(d s, d s)_{s(x)} \in T_{x}^{*} M \otimes T_{x}^{*} M$. We say $s$ is harmonic with respect to $\mu$ if $s$ is a critical point of the energy integral

$$
E(s, \mu)=\int_{M} \operatorname{trace} I(s) * 1
$$

Here trace and * can be taken in any metric compatible with $\mu$. We say that a branched immersion $s \in C^{\infty}(M, N)$ (see [10] for a definition) is a branched minimal immersion if $s$ is a critical point of the area integral

$$
A(s)=\int_{M}(\operatorname{det} I(s))^{1 / 2} * 1 .
$$

We recall the following relations between the two integrals. Theorem 1.2 lies at the basis of our method. 
THEOREM 1.1. Let $s: M \rightarrow N$ be a conformal branched immersion with respect to the conformal structure $\mu$ on $M$. Then $s$ is harmonic with respect to $\mu$ if and only if $s$ is a branched minimal immersion.

THEOREM 1.2 [18, THEOREM 1.8]. Let $s: M \rightarrow N$ be harmonic with respect to the conformal structure $\mu$ and suppose that $\mu$ is a critical point of $E(s, \cdot)$ with respect to all $C^{\infty}$ variations of $\mu$. Then $s$ is a conformal branched minimal immersion of $M$ into $N$.

The next theorem is the basic existence theorem we shall be using to get minimal immersions of Riemann surfaces into certain compact Riemannian manifolds. For a fixed conformal structure $\mu$ on $M$, let $E(s)=E(s, \mu)$. We shall say that $s: M \rightarrow N$ has the same action on $\pi_{1}(M)$ as $u: M \rightarrow N$ if, for a fixed $z \in M$ with

$$
s_{*}: \pi_{1}(M, z) \rightarrow \pi_{1}(N, s(z)) \text { and } u_{*}: \pi_{1}(M, z) \rightarrow \pi_{1}(N, u(z)),
$$

there exists a path $\alpha$ from $s(z)$ to $u(z)$ such that $\alpha_{*}$ conjugates $\pi_{1}(N, s(z))$ to $\pi_{1}(N, u(z))$, i.e., $s_{*}=\alpha_{*} \circ u_{*} \circ \alpha_{*}^{-1}$.

Theorem 1.3 (Theorem 5.2 of [18] AND TheOREM 2.1 of [17]). Let $u: M \rightarrow N$ be continuous. Then there exists a harmonic map $s: M \rightarrow N$ with the same action on $\pi_{1}(M)$ as $u$. Moreover, $s$ may be chosen to take on the minimum of the energy integral among all such maps.

Theorem 1.3 implies the following generalization (for domain manifolds of dimension two) of the well-known result of Eells and Sampson (see [8]).

Corollary 1.4 (TheOREM 5.1 OF [18]; SEE Also [15]). If $\pi_{2}(N)=0$, in particular, if $N$ has nonpositive sectional curvature, then there exists a minimizing harmonic map in every homotopy class of maps in $C^{0}(M, N)$.

COROLlaRY 1.5. If the map s of Theorem 1.3 is also a minimum of $E$ with respect to all smooth variations of $\mu$ preserving the action on $\pi_{1}(M)$, then $A(s) \leqslant A\left(s^{\prime}\right)$ for all branched immersions $s^{\prime}$ having the same action on $\pi_{1}(M)$ as $u$.

Proof. Suppose that $A\left(s^{\prime}\right)<A(s)$ for some $s^{\prime}$ as in the statement. Then the immersion $s^{\prime}$ induces a conformal structure on $M$, and $2 A\left(s^{\prime}\right)=E\left(s^{\prime}\right)$ for $E$ defined by this conformal structure. Let $\tilde{s}: M \rightarrow N$ be the minimizing map for this conformal structure with the same action on $\pi_{1}(M)$ as $u$. Then $E(\tilde{s}) \leqslant E\left(s^{\prime}\right)=2 A\left(s^{\prime}\right)<2 A(s)$ $=E(s)$, a contradiction.

Finally, in examining the behaviour of the energy integral near boundary points of Teichmüller space, the following extension theorem will be used.

THEOREM 1.6. Let $D$ denote the open unit disk in the complex plane. If $s$ : $D-\{0\} \rightarrow N$ is harmonic with finite energy, then s extends to a $C^{\infty}$ harmonic map $s:$ $D \rightarrow N$ (see [18, Theorem 3.6]).

2. Convergence of sequences of harmonic maps. In $\S \S 3$ and 4 we shall need to examine the convergence of certain sequences of harmonic maps. In this section we state the necessary convergence theorems. Their proofs are identical to those of similar theorems in $\S 4$ of [18]. The only (inessential) difference is that in the present 
context we deal only with harmonic maps rather than with the critical maps of a perturbed energy integral, i.e., the numbers $\alpha \geqslant 1$ of [18] are always taken to equal one. For $\mathscr{Q} \subset M$ any open set we denote by $L_{1}^{2}\left(\mathscr{Q l}, \mathbf{R}^{k}\right)$ the usual Sobolev space of functions which are in $L^{2}$ and whose (weak) first derivatives are also in $L^{2}$.

Lemma 2.1 [18, Theorem 4.1]. Let $s_{i}:$ Q $\rightarrow \rightarrow N$ be a sequence of harmonic maps with $E\left(s_{i}\right)<B$ for $i=1,2, \ldots$ Then there exists a subsequence $\left\{s_{i}^{\prime}\right\} \subset\left\{s_{i}\right\}$ such that $s_{i}^{\prime}$ converges to a limit $s$ weakly in $L_{1}^{2}\left(\vartheta_{1}, \mathbf{R}^{k}\right)$ and $\lim _{i \rightarrow \infty} E\left(s_{i}^{\prime}\right) \geqslant E(s)$.

Theorem 2.2 [18, TheOREM 4.4]. Let $s_{i}:$ Ql $\rightarrow N$ be a sequence of harmonic maps such that $E\left(s_{i}\right)<B$ for $i=1,2, \ldots$, and $s_{i}$ converges weakly in $L_{1}^{2}\left(\mathscr{Q}, \mathbf{R}^{k}\right)$. Then there exists a subsequence $\left\{s_{i}^{\prime}\right\} \subset\left\{s_{i}\right\}$ and a finite set of points $z_{1}, \ldots, z_{q}$ in $M$ such that $s_{i}^{\prime}$ converges to $s$ in $C^{1}\left(\vartheta \mathrm{l}-\left\{z_{1}, \ldots, z_{q}\right\}, N\right)$ and $s$ : $\vartheta 1 \rightarrow N$ is a $C^{\infty}$ harmonic map. Moreover, $E(s) \leqslant \liminf _{i \rightarrow \infty} E\left(s_{i}^{\prime}\right)$.

We need an extension of Theorem 2.2 in which the metric on $M$ is varied. Assume that a convergent sequence $\mu_{i} \rightarrow \mu$ of conformal structures on $M$ is given, corresponding to a sequence of metrics converging in the $C^{\infty}$ topology.

THEOREM 2.3. Let $s_{i}: M \rightarrow N$ be harmonic for the conformal structure $\mu_{i}$ on $M$, with $\mu_{i} \rightarrow \mu$ and $E\left(s_{i}, \mu_{i}\right) \leqslant B$. Then there exists a subsequence $\left\{s_{i}^{\prime}\right\} \subset\left\{s_{i}\right\}$ which converges to a harmonic map $s$ in $C^{\prime}\left(M-\left\{z_{1}, \ldots, z_{q}\right\}, N\right)$ and $E(s, \mu) \leqslant \liminf _{i \rightarrow \infty} E\left(s_{i}^{\prime}, \mu_{i}\right)$.

Proof. Cover $M$ with a finite set of coordinate charts $\mathcal{Q}_{j}$, each mapped conformally onto the open unit disk in the complex plane and each isothermal with respect to the conformal structure $\mu$ on $M$. Let $\mathscr{q}_{j, i}$ denote the coordinate chart $\mathscr{Q}_{j}$ provided with the conformal structure $\mu_{i}$. By the Riemann mapping theorem there exist conformal maps $\rho_{j, i}: \mathcal{Q}_{j, i} \rightarrow \mathcal{Q}_{j}$ fixing three given points. Since $\mu_{i} \rightarrow \mu$, we may assume that $\rho_{j, i}$ converges to the identity in $\operatorname{Diff}^{1}\left(\theta l_{j}\right)$.

Since $\mu_{i} \rightarrow \mu$, we can assume that $\left\{s_{i}\right\} \subset L_{1}^{2}\left(M, \mathbf{R}^{k}\right)$ is weakly convergent with respect to the conformal structure $\mu$ on $M$. Then $s_{i}^{\circ} \rho_{j, i}^{-1}: \mathcal{Q}_{j} \rightarrow N$ is a sequence of harmonic maps satisfying the hypothesis of Theorem 2.2. Thus a subsequence $\left\{s_{i}^{\prime}\right\}$ exists such that $s_{i}^{\prime} \circ \rho_{j, i}^{-1}$ converges in $C^{1}\left(\mathcal{Q l}_{j}-\left\{z_{1}, \ldots, z_{q}\right\}, N\right)$. Finally, since $M=\cup_{j} \vartheta_{1}$, we get the result on all of $M$.

We note in passing that for $i$ sufficiently large, $s_{i}^{\prime}$ and $s$ must induce the same action on $\pi_{1}(M)$, since one can simply choose representative loops generating $\pi_{1}(M)$ which avoid the points $\left\{z_{1}, \ldots, z_{q}\right\}$.

3. Minimal immersions of tori. We prove our theorems separately in the case of the torus for two reasons. Firstly, the moduli space for conformal structures on the torus is familiar, and the proofs can be done without any appeal to the more complicated theory of moduli spaces for Riemann surfaces of higher genus. Secondly, since the universal covering space of the torus is the plane, and not the open unit disk, there is a technical difference in the proofs, although there is none in the statement of the theorems.

The moduli space for conformal structures on tori is the upper-half plane $U$, which is the Teichmüller space for closed surfaces of genus one (for a definition of 
Teichmüller space see $\S 4)$. We identify $\xi \in U$ with the torus

$$
M_{\xi}=\mathbf{R}^{2} /\{z=z+1 \text { and } z=z+\xi\} .
$$

Here two generators of $\pi_{1}\left(S^{1} \times S^{1}\right)$ have been distinguished. If we do not distinguish generators, two elements $\xi_{1}, \xi_{2} \in U$ represent conformally equivalent tori if $\xi_{2}=\tau \xi_{1}$ for some $\tau \in \operatorname{PSL}(2, \mathbf{Z})=\operatorname{SL}(2, \mathbf{Z}) /\{ \pm I\}$, the modular group of the torus.

For $\xi \in U$, let $E(s, \xi)$ denote $E(s)$ defined on the torus $M_{\xi}$. Given $u: S^{1} \times S^{1} \rightarrow N$, define

$$
\tilde{E}(\xi)=\min \left\{E(s, \xi): s \text { has the same action on } \pi_{1}(M) \text { as } u\right\} \text {. }
$$

LEMMA 3.1. There exists a harmonic map $s: M_{\xi} \rightarrow N$ with the same action on $\pi_{1}(M)$ as $u$ for each $\xi \in U$. Moreover, $\tilde{E}$ is a continuous function on $U$.

Proof. The existence of $s$ follows from Corollary 1.4. The continuity of $E$ implies that of $\tilde{E}$.

THEOREM 3.2. Let $N$ be a compact Riemannian manifold and let $u$ : $S^{1} \times S^{1} \rightarrow N$ be such that $u_{*}: \pi_{1}\left(S^{1} \times S^{1}, z\right) \rightarrow \pi_{1}(N, u(z))$ (here $z$ is a base point on $\left.S^{1} \times S^{1}\right)$ contains no generators of $\pi_{1}\left(S^{1} \times S^{1}, z\right)$ in its kernel. Then if $\xi_{i} \in U$ is a sequence such that $\tilde{E}\left(\xi_{i}\right) \leqslant B$, there exist elements $\tau_{i} \in \operatorname{PSL}(2, \mathbf{Z})$ and a bound $b(B)$ depending only on $B$ such that $\tau_{i} \xi_{i}$ lies in the fundamental domain of $\operatorname{PSL}(2, \mathbf{Z})$ and $\operatorname{Im}\left(\tau_{i} \xi_{i}\right) \leqslant$ $b(B)$.

Proof. The existence of $\tau_{i}$ follows from the definition of fundamental domain.

Suppose there is a sequence $\xi_{i}$ such that $\kappa_{i}=\operatorname{Im}\left(\tau_{i} \xi_{i}\right)$. Let $\eta_{i}=\tau_{i} \xi_{i}$. By Lemma 3.1 there exist harmonic $s_{\xi_{i}}: M_{\xi_{i}} \rightarrow N$ with the same action on $\pi_{1}(M)$ as $u$. Then

$$
s_{i}=s_{\xi_{1}} \circ \tau_{i}^{-1}: M_{\eta_{i}} \rightarrow N
$$

is harmonic and $E\left(s_{i}, \eta_{i}\right)=E\left(s_{\xi_{i}}, \xi_{i}\right)=\tilde{E}\left(\xi_{i}\right) \leqslant B$.

Let $\bar{s}_{i}$ be the restriction of $s_{i}$ to $S^{1} \times\left[-\kappa_{i} / 4, \kappa_{i} / 4\right]$. Then $E\left(\bar{s}_{i}\right) \leqslant E\left(s_{i}, \eta_{i}\right) \leqslant B$. By Theorem 2.2, on any cylinder $S^{1} \times(-\kappa, \kappa)$ we can find a subsequence of $s_{i} \mid S^{1} \times$ $(-\kappa, \kappa)$ which converges in $C^{1}\left(S^{1} \times(-\kappa, \kappa)-\left\{z_{1}, \ldots, z_{q}\right\}, N\right)$ to a harmonic map $\bar{s}$ : $S^{1} \times(-\kappa, \kappa) \rightarrow N$ with $E(\bar{s}) \leqslant B$. Since $\kappa$ is arbitrary, $\bar{s}: S^{1} \times \mathbf{R} \simeq S^{2}-\left\{p_{1}, p_{2}\right\} \rightarrow$ $N$ is harmonic with $E(\bar{s}) \rightarrow B$. By Theorem 1.6, $\bar{s}$ extends to a $C^{\infty}$ map $s: S^{2} \rightarrow N$, providing a homotopy of $\bar{s}_{i}\left(S^{1} \times\{\alpha\}\right) \simeq \bar{s}\left(S^{1} \times\{\alpha\}\right)$ to a point for suitable $\alpha$. This implies that the generator $\tau_{i}^{-1}\left(S^{1} \times\{\alpha\}\right)$ of $M_{\xi_{i}}$ is mapped by $\bar{s}_{i}$, and hence also by $u$, to a loop homotopic to zero, which contradicts the assumption on the kernel of $u_{*}$.

THEOREM 3.3. Let $N$ be a compact Riemannian manifold of dimension $\geqslant 3$ and let $u$ : $S^{1} \times S^{1} \rightarrow N$ be such that $u_{*}: \pi_{1}\left(S^{1} \times S^{1}, z\right) \rightarrow \pi_{1}(N, u(z))$ has no generators of $\pi_{1}\left(S^{1} \times S^{1}, z\right)$ in its kernel. Then for some $\xi \in U$ there exists a conformal branched minimal immersion $s: M_{\xi} \rightarrow N$ with least area among branched immersions with the same action on $\pi_{1}(M)$ as $u$.

Proof. By Theorem 1.2, Corollary 1.5 and Lemma 3.1 we need only find a minimum of $\tilde{E}$ on $U$. Let $\xi_{i} \in U$ be such that $\lim _{i \rightarrow \infty} \tilde{E}\left(\xi_{i}\right)=\inf \{\tilde{E}(\xi): \xi \in U\}$. We 
may assume that $\tilde{E}\left(\xi_{i}\right) \leqslant B$ uniformly in $i$. As in the proof of Theorem 3.2 we can obtain harmonic maps $s_{i}: M_{\eta_{i}} \rightarrow N$ with $\operatorname{Im}\left(\eta_{i}\right) \leqslant b(B)$ and such that $s_{i}$ has the same action on $\pi_{1}(M)$ as $u \circ \tau_{i}^{-1}$. Choose a subsequence of $\left\{\eta_{i}\right\}$ which converges to $\eta$. By Theorem 2.3, we may pass to a further subsequence $s_{i}^{\prime}$ which converges to $s$ in $C^{1}\left(M_{\eta}-\left\{z_{1}, \ldots, z_{q}\right\}, N\right)$ with $s$ harmonic and $E(s, \eta) \leqslant \liminf _{i \rightarrow \infty} E\left(s_{i}^{\prime}, \eta_{i}\right)=$ $\lim _{i \rightarrow \infty} \tilde{E}\left(\xi_{i}\right)$. Since convergence in $C^{1}$ fails possibly only at the points $z_{j}$, it follows that $s_{i}=s_{\xi_{1}} \circ \tau_{i}^{-1}$ has the same action on $\pi_{1}(M)$ as $s$ for $i$ sufficiently large. Thus $s \circ \tau_{i}$ is a harmonic map from $M_{\tau_{i}(\eta)}$ into $N$ which has the same action on $\pi_{1}(M)$ as $u$, and such that $\lim _{i \rightarrow \infty} E\left(s \circ \tau_{i}, \tau_{i}^{-1}(\eta)\right)=\lim _{i \rightarrow \infty} \tilde{E}\left(\xi_{i}\right)$.

4. Minimal immersions of surfaces of genus larger than one. The technique for obtaining our main theorem in this section, Theorem 4.4 , will be to use the dependence of $E$ on conformal structure and Theorem 1.2 to transfer our variational problem to a suitable space of moduli for conformal structures on a surface of fixed genus. We simply state the necessary results about moduli spaces for Riemann surfaces and refer the reader to Bers [4] for general background and to Abikoff [1, 2, 3] and Bers [5, 6, 7] for the theory of the boundaries of these spaces.

Given a closed Riemann surface $M$ of genus larger than one, one can define the Teichmüller space $\mathcal{T}(M)$ to be the space of all equivalence classes of pairs $\left(M^{\prime}, \alpha\right)$, where $\alpha$ is a quasi-conformal bijection from $M$ onto $M^{\prime}$. Two pairs $\left(M^{\prime}, \alpha_{1}\right)$ and $\left(M^{\prime \prime}, \alpha_{2}\right)$ are equivalent if and only if $\alpha_{2} \circ \alpha_{1}^{-1}$ is homotopic to a conformal map. If we "mark" $M$ by choosing a set of simple closed curves on $M$ whose homotopy classes generate $\pi_{1}(M)$, then $\mathscr{T}(M)$ can be considered as the space of all equivalence classes of marked Riemann surfaces. The mapping class group of $M, \Gamma^{+}(M)$, is the group of all orientation-preserving homeomorphisms of $M$ modulo the subgroup of homeomorphisms isotopic to the identity. The Teichmüller modular group of $M$, denoted $\operatorname{Mod}(M)$, is the mapping class group of $M$ modulo its subgroup of ineffective elements. $\operatorname{Mod}(M)$ acts on $T(M)$ by pulling back conformal structures. If $\tau \in \operatorname{Mod}(M)$ and $M^{\prime \prime}=\tau\left(M^{\prime}\right)$ as a Riemann surface, then $M^{\prime}$ and $M^{\prime \prime}$ are conformally equivalent but have different markings.

A Riemann surface with nodes is a connected complex space $M$ such that every point $z$ on $M$ has a neighbourhood isomorphic either to the open unit disk (with $z$ corresponding to the center), or to two unit disks, with centers identified and corresponding to $z$. Riemann surfaces with nodes are obtained by passing to the Bers boundary of Teichmüller space (the latter is diffeomorphic to an open cell of real dimension $6 g-6, g=$ genus of $M$ ) by "pinching" some set of admissible simple closed curves on $M$ to nodes. See Bers $[5,6]$.

Let $R(M)=T(M) / \operatorname{Mod}(M)$. Then $R(M)$ is the classical Riemann moduli space for conformal structures on $M$. The following result is contained in Abikoff [1].

THEOREM 4.1. There exists a compactification $\hat{R}(M)$ of $R(M)$ such that the boundary points $\hat{R}(M)-R(M)$ correspond precisely to the Riemann surfaces with nodes that can be obtained from $M$ by "pinching" a set of admissible closed curves on $M$. 
$\hat{R}(M)$ is called the augmented Riemann space for the genus of $M$. When genus $M=1, \mathcal{T}(M)$ is the upper half-plane $U$ and $\operatorname{Mod}(M)=\operatorname{PSL}(2, \mathbf{Z}) . \hat{R}(M)$ is then obtained by compactifying $U / \operatorname{PSL}(2, \mathbf{Z})$ by adding one point at infinity so that $\hat{R}(M)$ is a sphere with a pair of nodes.

Now let $M_{0}$ be an arbitrarily chosen fixed Riemann surface of genus $g \geqslant 2$, and let $M_{0}$ be a base surface for the Teichmüller space $\mathcal{T}\left(M_{0}\right)$ for surfaces of genus $g$. Since $E$ is conformally invariant we can define an energy integral on $\mathscr{T}\left(M_{0}\right)$ as follows: Let $M_{\mu}$ denote the marked Riemann surface represented by the point $\mu \in \mathcal{T}\left(M_{0}\right)$. Define, for a fixed map $u: M \rightarrow N, \tilde{E}(\mu)=\inf \{E(s, \mu): s$ has the same action on $\pi_{1}(M)$ as $\left.u\right\}$.

LEMMA 4.2. $\tilde{E}(\mu)$ is attained on each $M_{\mu}$ by some $s_{\mu}$ which has the same action on $\pi_{1}(M)$ as $u$, and $\tilde{E}$ is continuous on $\mathcal{T}\left(M_{0}\right)$.

Proof. The existence of $s_{\mu}$ follows from Corollary 1.4. The continuity of $\tilde{E}$ follows from that of $E$.

THEOREM 4.3. Let $s_{i}: M_{\mu_{i}} \rightarrow N$ be a sequence of maps harmonic in the conformal structure $\mu_{i}$ on $M$ with $E\left(s_{i}, \mu_{i}\right) \leqslant B$. Suppose also that $\pi\left(M_{\mu_{i}}\right) \rightarrow M_{\infty}$ where $\pi$ : $\mathcal{T}\left(M_{0}\right) \rightarrow R\left(M_{0}\right)$ is the quotient map and $M_{\infty} \in \hat{R}\left(M_{0}\right)-R\left(M_{0}\right)$. Then for $i$ sufficiently large, $\left(s_{i}\right)_{*}(\gamma)=1$ for at least one $\gamma \in \pi_{1}(M)$ represented by a simple closed curve.

Proof. The sequence $M_{\mu_{i}}$ provides a deformation of $M_{0}$ onto a Riemann surface with nodes $M_{\infty}$ corresponding to "pinching" a set of homotopically nontrivial simple closed curves $\gamma_{m}$ to nodes $w_{m} ; m=1, \ldots, n$. A sequence $\left\{D_{j}^{m}\right\}$ of closed annular neighbourhoods of $\gamma_{m}$ can be chosen so that $D_{j}^{m}$ converges to the node $w_{m}$ of $M_{\infty}$ and, for each fixed $j$, the change in conformal structure on $M_{0}$ as $M_{\mu_{i}} \rightarrow M_{\infty}$ is restricted to the interior of $\cup_{m} D_{j}^{m}$ (see Bers [5]). Let $M_{j}=M_{0}-\cup_{m} D_{j}^{m}$. Then, for fixed $j,\left\{s_{i} \mid M_{j}\right\}$ is a sequence of harmonic maps on $M_{j}$ which by Theorem 2.3 converges in $C^{1}\left(M_{j}-\left\{z_{1}, \ldots, z_{q}\right\}, N\right)$ to a smooth harmonic map $s^{(j)}: M_{j} \rightarrow N$. By the unique continuation property for harmonic maps (see [12]), $s^{(k)}$ extends $s^{(j)}$ from $M_{j}$ to $M_{k}$ for each $k>j$. Now let $j \rightarrow \infty$ to get a smooth harmonic map $s: M_{\infty}^{\prime} \rightarrow N$, where $M_{\infty}^{\prime}$ is the punctured Riemann surface $M_{0}-\left\{w_{1}, \ldots, w_{n}\right\}$ and $s^{(j)} \rightarrow s$ in $C^{\prime}\left(M_{\infty}^{\prime}-\left\{z_{1}, \ldots, z_{q}\right\}, N\right)$. Since $E\left(s_{i}\right) \leqslant B$ for all $i, E(s) \leqslant B$. By Theorem 1.6, $s$ can be extended to a smooth harmonic map $\tilde{s}: \tilde{M}_{\infty} \rightarrow N$, where $\tilde{M}_{\infty}=M_{\infty}^{\prime} \cup$ $\left\{\left(x_{1}, x_{1}^{\prime}\right), \ldots,\left(x_{n}, x_{n}^{\prime}\right)\right\}$ is the closed Riemann surface obtained by adding a pair of points $\left(x_{m}, x_{m}^{\prime}\right)$ at the two punctures of $M_{\infty}^{\prime}$ corresponding to each node $w_{m} \in M_{\infty}$.

Choose any curve $\gamma$ homotopic to $\gamma_{m}$, for any fixed $m$ between 1 and $n$. Clearly, $\gamma$ can be chosen so as not to contain any of the points $z_{1}, \ldots, z_{q}$ and to lie in $D_{j}^{m}$ for some large $j$. Since $\gamma \subset \tilde{M}_{\infty}$ is homotopically trivial, it follows that $s(\gamma)$ is homotopically trivial. But $\lim _{i \rightarrow \infty} s_{i}(\gamma)=s(\gamma)$, so that $s_{i}(\gamma)$ is homotopically trivial for $i$ sufficiently large. 
THEOREM 4.4. Let $M$ be a closed topological surface of genus larger than one and let $u: M \rightarrow N$ be a continuous map such that $u_{*}: \pi_{1}(M) \rightarrow \pi_{1}(N)$ contains no nontrivial element represented by a simple closed curve in its kernel. Then there exists a conformal branched minimal immersion $s: M \rightarrow N$ with the same action on $\pi_{1}(M)$ as u such that $A(s) \leqslant A\left(s^{\prime}\right)$, for all branched immersions $s^{\prime}: M \rightarrow N$ with the same action on $\pi_{1}(M)$ as $u$. If $\pi_{2}(N)=0$, then $s$ can be chosen homotopic to $u$.

Proof. By Theorem 1.2, Corollary 1.5 and Lemma 4.2, it is sufficient to show that a minimum of $\tilde{E}$ is attained at some point in $T(M)$. Let $\xi_{i} \in \widetilde{T}(M)$ be a minimizing sequence for $\tilde{E}$. By Lemma 4 of Abikoff [1], there is a subsequence of $\left\{\xi_{i}\right\}$ (denoted again by $\left.\left\{\xi_{i}\right\}\right)$ and elements $\tau_{i} \in \operatorname{Mod}(M)$ such that $\mu_{i}=\tau_{i}\left(\xi_{i}\right)$ either converges to a limit in $T(M)$ or $M_{\mu_{t}}$ converges to a surface with nodes. Let $s_{i}$ be a minimizing harmonic map in the homotopy class of $u \circ \tau_{i}^{-1}$ relative to the conformal structure $\mu_{i}=\tau_{i}\left(\xi_{i}\right)$. Note that $E\left(s_{i}, \mu_{i}\right)=\tilde{E}\left(\xi_{i}\right)$ is bounded. Therefore, since no nontrivial simple loop in $M$ maps onto a homotopically trivial loop in $N$, Theorem 4.3 implies that $\lim _{i \rightarrow x} \mu_{i}=\mu \in \mathcal{T}(M)$. By Theorem 2.3, there is a subsequence $\left\{s_{i}^{\prime}\right\} \subset\left\{s_{i}\right\}$ such that $s_{i}^{\prime} \rightarrow s$ in $C^{1}\left(M-\left\{z_{1}, \ldots, z_{q}\right\}, N\right), s$ harmonic with respect to $\mu$ and $\liminf _{i \rightarrow \infty} E\left(s_{i}^{\prime}, \mu_{i}\right)=\liminf _{i \rightarrow \infty} \tilde{E}\left(\xi_{i}\right) \geqslant E(s, \mu)$. Since the convergence of $s_{i}$ to $s$ is valid away from the isolated points $z_{j}, s$ has the same action on $\pi_{1}(M)$ as $s_{i}$ for $i$ sufficiently large and therefore $s \circ \tau_{i}$ has the same action on $\pi_{1}(M)$ as $u$. Finally, the inequalities

$$
\tilde{E}\left(\tau_{i}^{-1}(\mu)\right) \leqslant E\left(s \circ \tau_{i}(\mu), \tau_{i}^{-1}(\mu)\right)=E(s, \mu) \leqslant \liminf _{i \rightarrow \infty} \tilde{E}\left(\xi_{i}\right)
$$

show that the minimum is taken on at $\tau_{i}^{-1}(\mu)$.

If $\pi_{2}(N)=0$, then by Corollary 1.4 each $s_{i}$ can be chosen homotopic to $u$, and a similar argument as before gives $s$ homotopic to $u$.

5. Morse theory. It has been known for some time that when $N$ has negative sectional curvature, harmonic maps into $N$ from a Riemannian manifold $M$ are essentially unique within their homotopy class [11]. The corresponding question for uniqueness within a homotopy class of minimal immersions of surfaces into an $N$ with negative sectional curvature has been open for some time. W. Thurston pointed out a counterexample which suggested to us a means of proving the nonuniqueness result of this section, Corollary 6.5, which is an application of Ljusternik-Schnirelman Theory and Morse Theory to situations similar to that of Thurston's example.

Both T. Jørgensen [14] and W. Thurston [20, 19] constructed compact hyperbolic 3-manifolds which fiber over $S^{1}$. Consider such a 3-manifold $N$ with fiber a closed orientable surface $M_{0}$ of genus larger than one. By Theorem 4.4 there exists a conformal branched minimal immersion of $M_{0}$ into $N$ in the homotopy class of any fixed inclusion map of $M_{0}$ in $N$. Let $\tilde{N} \rightarrow N$ be the double covering of $N$ corresponding to the subgroup of $\pi_{1}(N)$ which maps onto even elements of $\pi_{1}\left(S^{1}\right)$ under the fibering of $N$ over $S^{1}$. Thurston pointed out that the two preimages $M_{1}$ and $M_{2}$ in $N$ of $M_{0}$ are homotopic minimal immersions. In this fashion we can construct any finite number of minimal immersions in the homotopy class of the inclusion map of the fiber. 
For the remainder of this section we shall assume that $N$ is compact with negative sectional curvature. We shall also assume that $u: M \rightarrow N$ is given and that $u_{*}$ : $\pi_{1}(M) \rightarrow \pi_{1}(N)$ is injective. Let $\tilde{E}: \mathcal{T}(M) \rightarrow \mathbf{R}^{+}$be as in $\S 4$.

THEOREM 5.1. There exists a unique $s_{\mu}$ homotopic to $u$ such that $\tilde{E}(\mu)=E\left(s_{\mu}, \mu\right)$. Moreover, $\tilde{E}$ is smooth as a function on $\mathcal{T}(M)$.

Proof. The existence of $s_{\mu}$ follows from Corollary 1.4 and Lemma 4.2. By the Cartan-Hadamard theorem, all maps homotopic to $u$ have the same action on $\pi_{1}(M)$ as $u$. The uniqueness of $s_{\mu}$ is a result of P. Hartman [11]. In this case, the critical points $s_{\mu}$ of $E$ are nondegenerate, and an application of the implicit function theorem proves that $s_{\mu}$ is a smooth function of $\mu \in \mathcal{T}(M)$ (see, for example, [21]). The smoothness of $\tilde{E}(\mu)=E\left(s_{\mu}, \mu\right)$ follows.

We observed earlier that if $\tau \in \operatorname{Mod}(M)$, then $E(s, \mu)=E(s, \tau(\mu))$, which suggests the possibility of reducing our variational problem to one on $R(M)=$ $\widetilde{T}(M) / \operatorname{Mod}(M)$, the Riemann moduli space for the genus of $M$. There are two obstructions to doing this. Firstly, $R(M)$ is not a manifold, but an analytic space, and secondly, $\operatorname{Mod}(M)$ does not leave the homotopy class $\{u\}$ invariant. However, there are subgroups of $\operatorname{Mod}(M)$ which, for certain $N$, do not present these difficulties.

Unfortunately, we cannot consider only the orientation-preserving diffeomorphisms of $M$. Let $\operatorname{Diff}_{0}(M)$ be the subgroup of $\operatorname{Diff}(M)$ consisting of diffeomorphisms isotopic to the identity. Then $\Gamma(M)=\operatorname{Diff}(M) / \operatorname{Diff}_{0}(M)$ is called the extended mapping class (or homeotopy) group of $M$. The mapping class group $\Gamma^{+}(M)=\operatorname{Diff}^{+}(M) / \operatorname{Diff}_{0}(M)$, where $\operatorname{Diff}^{+}(M)$ are the orientation-preserving diffeomorphisms of $M$. $\Gamma^{+}(M)$ has index two in $\Gamma(M)$. Both $\Gamma^{+}(M)$ and $\Gamma(M)$ act in a natural way on $\mathcal{T}(M)$ by pulling back conformal structures. If $M$ has genus two, there exist ineffective elements of $\Gamma^{+}(M)$, accounting for the only difference between $\Gamma^{+}(M)$ and $\operatorname{Mod}(M)$.

For $u: M \rightarrow N$ such that $u_{*}: \pi_{1}(M) \rightarrow \pi_{1}(N)$ is an injection, define the subgroup $K \subset \Gamma(M)$ by

$$
K=K(u)=\{\tau \in \Gamma(M): u \circ \tau \text { is homotopic to } u\}
$$

First we shall show how this group fits into the Morse Theory and then we shall describe the possible $K$ for certain 3-manifolds $N$.

Proposition 5.2. $\tilde{E}: \mathcal{T}(M) \rightarrow \mathbf{R}^{+}$is the lift of a function $\hat{E}: \mathcal{T}(M) / K \rightarrow \mathbf{R}^{+}$.

Proof. We need only show that $\tilde{E}(\mu)=\tilde{E}(\tau(\mu))$ for any $\tau \in K$. Since $\left\{u \circ \tau^{-1}\right\}$ $=\{u\}$,

$$
\begin{aligned}
\tilde{E}(\mu) & =\min \{E(s, \mu): s \in\{u\}\}=\min \left\{E\left(s \circ \tau, \tau^{-1}(\mu)\right): s \in\{u\}\right\} \\
& =\min \left\{E\left(s, \tau^{-1}(\mu)\right): s \in\left\{u \circ \tau^{-1}\right\}\right\}=\tilde{E}\left(\tau^{-1}(\mu)\right)=\tilde{E}(\tau(\mu))
\end{aligned}
$$

THEOREM 5.3. The map $\hat{E}$ is proper on $\mathcal{T}(M) / K$. 
Proof. The proof is a variation of the proofs of Theorems 4.3 and 4.4. Let $S=E^{-1}[0, B]$. We show that $S / K$ is sequentially compact. Given $\left\{\xi_{i}\right\} \subset S$, by passing to a subsequence if necessary, we may assume that there exist $\tau_{i} \in \operatorname{Mod}(M)$ such that $\mu_{i}=\tau_{i}\left(\xi_{i}\right)$ converges to $\mu \in \mathcal{T}(M)$. Let $s_{i}$ be the unique harmonic map in the homotopy class $\left\{u \circ \tau_{i}^{-1}\right\}$ with respect to the conformal structure $\mu_{i}$. Pass again to a subsequence if necessary and get $s_{i} \rightarrow s$ in $C^{1}\left(M-\left\{z_{1}, \ldots, z_{q}\right\}, N\right), s: M_{\mu} \rightarrow N$ harmonic and $\left\{s \circ \tau_{i}\right\}=\{u\}$ for $i$ sufficiently large. It follows that $\tau_{j}^{-1} \circ \tau_{i}=\kappa_{i j} \in K$ for $i$ and $j$ sufficiently large. Here the difference between $\operatorname{Mod}(M)$ and $\Gamma^{+}(M)$ is of finite index and is not relevant to this proof. For fixed $\tau_{j}, \tau_{j}^{-1}\left(\mu_{i}\right) \rightarrow \tau_{j}^{-1}(\mu)=\xi$, say. But $\kappa_{i j}\left(\xi_{i}\right)=\tau_{j}^{-1} \circ \tau_{i}\left(\xi_{i}\right)=\tau_{j}^{-1}\left(\mu_{i}\right) \rightarrow \xi$ for $\kappa_{i j} \in K$, so that $S / K$ is sequentially compact.

THEOREM 5.4. If the elements of $K \subset \Gamma(M)$ act without fixed points, then $\hat{E}$ is a smooth proper map on $\widetilde{T}(M) / K$. Every critical point of $\hat{E}$ corresponds to exactly one branched minimal immersion of $M$ into $N$ homotopic to $u$ and conversely, every geometric unramified branched minimal immersion of $M$ into $N$ homotopic to $u$ corresponds to exactly one critical point of $\hat{E}$.

Proof. $\mathscr{T}(M) / K$ is a manifold since all the elements of $K$ are assumed to act without fixed points. By Theorem 1.2, every critical point of $\hat{E}$ corresponds to a conformal branched minimal immersion of $M$ into $N$. We need to show that every geometric unramified branched minimal immersion corresponds to only one critical point of $\hat{E}$.

Suppose $s_{1}: M \rightarrow N$ and $s_{2}: M \rightarrow N$ are both branched minimal immersions homotopic to $u$, and $s_{1}(M)=s_{2}(M)$. We need to show that $s_{2}=s_{1} \circ \tau$ for some $\tau \in \Gamma(M)$. By a theorem of R. Gulliver, R. Osserman and H. Royden (3.12 of [10]), if $s_{1}$ is unramified, then no two disjoint open disks in $M$ are isomorphic to the same disk in $N$ under the immersion $s_{1}$. Define $\tau: M \rightarrow M$ as follows: $\tau(x)=\{y \in$ $s_{1}^{-1}\left(s_{2}(x)\right): s_{2}\left(\mathcal{Q}_{y}\right) \cap s_{1}\left(\mathcal{Q}_{x}\right)$ is an open set for every pair of neighbourhoods $\mathcal{Q l}_{y}$ of $y$ and $\mathscr{U}_{x}$ of $x$ \}. Then $\tau(x)$ is nonempty because $s_{1}^{-1}\left(s_{2}(x)\right)$ is a nonempty finite set and $s_{1}$ and $s_{2}$ are immersions away from branch points. The hypothesis that $s_{1}$ is unramified ensures that $\tau(x)$ is unique and that $\tau$ is continuous. Let $\mu_{1}$ and $\mu_{2}$ be the conformal structures induced by $s_{1}(M) \subset N$ and $s_{2}(M) \subset N$, respectively. Then $\tau$ : $M_{\mu_{2}} \rightarrow M_{\mu_{1}}$ is conformal away from the branch points of $s_{1}$ and $s_{2}$. Since $\tau$ is either holomorphic or antiholomorphic, these singularities are removable and $\tau$ is conformal. By the same argument, $\tau^{-1}$ is conformal. It follows that $s_{2}=s_{1} \circ \tau$.

We now ask whether there are examples where $K$ acts without fixed points and T $(M) / K$ is an interesting space. The hyperbolic 3-manifolds of Jørgensen and Thurston, which fiber over the circle, provide essentially all the interesting examples (for a justification of this statement see §6). These 3-manifolds are the mapping tori for certain diffeomorphisms $\tau \in \Gamma(M)$. Namely,

$$
N=M \times \mathbf{R} /\{(x, t)=(\tau(x), t+1)\}
$$

as a differentiable manifold. If $i: M \hookrightarrow N$ is an inclusion of the fiber, then $K \simeq \mathbf{Z} \simeq$ $\left\{\tau^{n} ; n\right.$ an integer $\}$. 
COROLlaRY 5.5. Let $N$ be a hyperbolic 3-manifold which fibers over $S^{1}$ and let $i$ : $M \hookrightarrow N$ be an inclusion of the fiber. Then there are at least two geometrically distinct minimal immersions of $M$ into $N$ which are homotopic to $i$.

Proof. Here $K=\mathbf{Z}$ and $K$ acts without fixed points on $\mathcal{T}(M)$, and there is no ramified immersion homotopic to $i$. By Theorem 5.4, the conformal branched minimal immersions of $M$ into $N$ homotopic to $i$ are in 1-to-1 correspondence with the critical points of the proper smooth function $\hat{E}: \mathcal{T}(M) / K \rightarrow \mathbf{R}^{+}$. Since $\mathcal{T}(M)$ is diffeomorphic to a cell, $\mathscr{T}(M) / K$ has the homotopy type of $S^{1}$ and therefore $\operatorname{cat}(T(M) / K)=\operatorname{cat}\left(S^{1}\right)=2$. Here cat denotes the Ljusternik-Schnirelman category. By Ljusternik-Schnirelman theory, every smooth proper map on $\mathscr{T}(M) / K$ has at least $\operatorname{cat}\left(S^{1}\right)=2$ critical points [16].

6. The subgroup $K \subset \Gamma(M)$. Given a map $u: M \rightarrow N$, we defined the subgroup $K=K(u) \subset \Gamma(M)$ as

$$
K=\{\tau \in \Gamma(M): u \circ \tau \in\{u\}\} .
$$

Note that $K$ depends only on $M, N$ and the homotopy class of $u$. In this section we give an alternative description of $K$ and work out some basic examples. We assume throughout the rest of this section that the following general properties hold:

(a) $u_{*}: \pi_{1}(M) \rightarrow \pi_{1}(N)$ is an injection. We shall identify $\pi_{1}(M)$ and its image $u_{*} \pi_{1}(M)$ in $\pi_{1}(N)$.

(b) $\pi_{1}(N)$ is torsion free.

(c) $N$ is atoral, i.e., $\pi_{1}(N)$ contains no subgroups isomorphic to $\mathbf{Z} \oplus \mathbf{Z}$.

(d) $\pi_{2}(N)=0$.

Note that if $N$ has negative sectional curvature, then (b)-(d) are automatically true.

Define $\tilde{K}$ as the stabilizer of $u_{*} \pi_{1}(M) \subset \pi_{1}(N)$ :

$$
\tilde{K}=\left\{\tilde{\alpha} \in \pi_{1}(N): \tilde{\alpha} \pi_{1}(M) \tilde{\alpha}^{-1}=\pi_{1}(M)\right\} .
$$

THEOREM 6.1. If (a)-(d) hold, then $K=\tilde{K} / \pi_{1}(M)$.

Proof. There is a natural homomorphism $j: \tilde{K} \rightarrow \Gamma(M)$. For $\tilde{\alpha} \in \tilde{K}$ and $\gamma \in$ $\pi_{1}(M)$, let $\tilde{\alpha}(\gamma)=\tilde{\alpha} \gamma \tilde{\alpha}^{-1}$. Thus $\tilde{\alpha}$ can be regarded as a map $\tilde{\alpha}: \pi_{1}(M) \rightarrow \pi_{1}(M)$. Since $M$ is a $K(\pi, 1)$, the map $\tilde{\alpha}: \pi_{1}(M) \rightarrow \pi_{1}(M)$ is induced by some $f_{\alpha}: M \rightarrow M$. Let $j(\tilde{\alpha})=\alpha$ be the projection of $f_{\alpha}$ into $\Gamma(M)$. We need to show that $\operatorname{ker} j=\pi_{1}(M)$ and that $j$ is surjective onto $K \subset \Gamma(M)$.

First note that $\pi_{1}(M) \subset \operatorname{ker} j$, for if $\tilde{\alpha} \in \pi_{1}(M)$, then the induced map $\tilde{\alpha}$ : $\pi_{1}(M) \rightarrow \pi_{1}(M)$ is an inner automorphism and $f_{\alpha}: M \rightarrow M$ is isotopic to 1. Conversely, if $j(\tilde{\alpha})=1$, then $\tilde{\alpha}: \pi_{1}(M) \rightarrow \pi_{1}(M)$ is an inner automorphism and $\tilde{\alpha} \gamma \tilde{\alpha}^{-1}=$ $\beta \gamma \beta^{-1}$ for all $\gamma \in \pi_{1}(M)$ and some $\beta \in \pi_{1}(M)$. It follows that $\left(\beta^{-1} \tilde{\alpha}\right) \gamma\left(\beta^{-1} \tilde{\alpha}\right)^{-1}=\gamma$ in $\pi_{1}(N)$ for all $\gamma \in \pi_{1}(M)$. Since $\pi_{1}(N)$ is atoral, this is impossible unless $\beta^{-1} \tilde{\alpha}=1$, i.e., $\beta=\tilde{\alpha} \in \pi_{1}(M)$. Thus ker $j=\pi_{1}(M)$.

To show that $j$ is surjective, assume that $\alpha \in K$ is represented by a basepoint preserving diffeomorphism $f_{\alpha}: M \rightarrow M$. Since $\alpha \in K$, there exists a homotopy $F$ : $M \times[0,1] \rightarrow N$ with $F(z, 0)=u(z)$ and $F(z, 1)=u\left(f_{\alpha}(z)\right)$. Let $\tilde{\alpha} \in \pi_{1}(N)$ represent 
the curve $F\left(z_{0}, t\right), 0 \leqslant t \leqslant 1$, where $z_{0}$ is the chosen basepoint of $M$, given by $\tilde{\alpha} \gamma \tilde{\alpha}^{-1}=\left(f_{\alpha}\right)_{*} \gamma$. Then $j(\tilde{\alpha})=\alpha$.

EXAMPLE 6.2. Let $\alpha \in \Gamma(M)$ have infinite order and be represented by a basepoint preserving map $f_{\alpha}: M \rightarrow M$ such that $\left(f_{\alpha}\right)_{*}: \pi_{1}(M) \rightarrow \pi_{1}(M)$ leaves no subgroup invariant. The mapping torus for $f_{\alpha}$ is the compact 3-manifold which fibers over $S^{1}$ obtained from $M \times \mathbf{R}$ by identifying $(x, t)$ with $\left(f_{\alpha}(x), t+1\right)$, i.e., $N=M$ $\times \mathbf{R} /\left\{(x, t)=\left(f_{\alpha}(x), t+1\right)\right\}$. Here $\pi_{1}(N)$ is generated by the subgroup $\pi_{1}(M)$ and the element $\tilde{\alpha}$ represented by the loop $\left(z_{0}, t\right)$, where $z_{0}$ is the chosen basepoint. The relations are $\tilde{\alpha} \gamma \tilde{\alpha}^{-1}=\left(f_{\alpha}\right)_{*} \gamma$, for $\gamma \in \pi_{1}(M)$. Let $u: M \rightarrow M \times \mathbf{R} \rightarrow N$ be in the homotopy class of an inclusion map of the fiber. Then $\tilde{K}=\pi_{1}(N)$ and $K \simeq$ $\tilde{K} / \pi_{1}(M) \simeq \mathbf{Z}$.

EXAMPLE 6.3. Let $\tilde{u}=u \circ h$ factor through a regular covering $h: \tilde{M} \rightarrow M$ where $u$ : $M \rightarrow N$ induces an injection on the fundamental group. Then the group of deck transformations $\pi_{1}(M) / \pi_{1}(\tilde{M})$ is a finite group which maps into $\Gamma(M)$. The image of $\pi_{1}(M) / \pi_{1}(\tilde{M})$ in $\Gamma(M)$ lies in $K$.

We assert that Examples 6.2 and 6.3 are essentially the only examples. Assume that $\alpha=j(\tilde{\alpha}) \in K$ is the image of $\tilde{\alpha} \in \tilde{K} \subset \pi_{1}(N)$ and let $\hat{K} \subset \tilde{K}$ be the subgroup generated by $\tilde{\alpha}$ and the image of $\pi_{1}(M)$ in $\pi_{1}(N)$. Example 6.3 describes what happens when $\alpha$ has finite order and Example 6.2 is typical of what happens when $\alpha$ has infinite order.

THEOREM 6.4. If $\alpha \in K$ has finite order $p$, then under the assumptions (a)-(d), $\tilde{u}$ : $\tilde{M} \rightarrow N$ is homotopic to $u \circ h$, where $h: \tilde{M} \rightarrow M$ is a regular p-sheeted covering and $u$ : $M \rightarrow N$.

Proof. Recall that by Nielsen theory [3, 7], $\alpha$ may be realized as a conformal map $f_{\alpha}: \tilde{M} \rightarrow \tilde{M}$ for some conformal structure on $\tilde{M}$. Let $Q$ be the subgroup of isometries (possibly orientation reversing) of the universal covering $H^{2}$ of $\tilde{M}$ generated by a lift $\tilde{f}_{\alpha}: H^{2} \rightarrow H^{2}$ of $f_{\alpha}: \tilde{M} \rightarrow \tilde{M}$ and the deck transformations $\pi_{1}(\tilde{M})$ on $H^{2}$. Up to inner automorphism, conjugation by $\tilde{f}_{\alpha}$ and $\tilde{\alpha}$ are the same on $\pi_{1}(\tilde{M})$. Thus, $\tilde{f}_{\alpha} \circ \gamma \circ \tilde{f}_{\alpha}^{-1}=$ $(\tilde{\alpha} x) \gamma(\tilde{\alpha} x)^{-1}$ for some $x \in \pi_{1}(\tilde{M})$ and all $\gamma \in \pi_{1}(\tilde{M})$. Note that $\tilde{\alpha} x \in \pi_{1}(N)$ has infinite order. Certainly $\tilde{f}_{\alpha}$ has infinite order, since $(\tilde{\alpha} x)^{p} \gamma(\tilde{\alpha} x)^{-p}=\gamma$ implies that $(\tilde{\alpha} x)^{p}=1$ also. Therefore, $\tilde{f}_{\alpha}$ is fixed-point free and $Q \simeq \hat{K}$ by identifying the subgroups $\pi_{1}(M) \subset Q$ with $\pi_{1}(\tilde{M}) \subset \hat{K}$ and $\tilde{f}_{\alpha}$ with $\tilde{\alpha} x$. It follows that $\tilde{M} \rightarrow \tilde{M} / f_{\alpha} \simeq$ $M$ is a regular covering with deck transformation group $\mathbf{Z}_{p} \simeq \hat{K} / \pi_{1}(\tilde{M})$. We know that $\tilde{u}_{*}: \pi_{1}(\tilde{M}) \rightarrow \pi_{1}(N)$ factors through a map into $\pi_{1}(M)$, that $\tilde{M}$ is a $K(\pi, 1)$ and $\pi_{2}(N)=0$. Hence, this factoring at the fundamental group level can be realized by a factoring on the space level.

Lemma 6.5. Suppose $\alpha \in K$ has infinite order, $\pi_{3}(N)=0$, and let $N_{\alpha}=M \times$ $\mathbf{R} /\left\{(x, t)=\left(f_{\alpha}(x), t+1\right)\right\}$. Then under the general assumptions $(\mathrm{a})-(\mathrm{d}), \pi_{1}\left(N_{\alpha}\right) \simeq \hat{K}$. Moreover, $u: M \rightarrow N$ is homotopic to a map $\tilde{u}: M \rightarrow N_{\alpha} \rightarrow N$, where $M \hookrightarrow N_{\alpha}$ is inclusion of the fiber and $N_{\alpha} \rightarrow N$ is some map. 
Proof. Observe that $\hat{K} / \pi_{1}(M) \simeq \pi_{1}\left(N_{\alpha}\right) / \pi_{1}(M) \simeq \mathbf{Z}$, and that conjugation by $\tilde{\alpha}$ in $\hat{K}$ agrees with conjugation represented by the loop $\left(z_{0}, t\right)$ in $N_{\alpha}$, and the groups have identical descriptions. The map $u_{*}: \pi_{1}(M) \rightarrow \pi_{1}(N)$ factors through $\pi_{1}(M) \rightarrow$ $\pi_{1}\left(N_{\alpha}\right) \rightarrow \pi_{1}(N)$. Since $M$ and $N_{\alpha}$ are $K(\pi, 1)$ 's and $\pi_{2}(N)=\pi_{3}(N)=0$, this factorization can be realized by a map on the space level.

THEOREM 6.6. Suppose that $N$ is a compact 3-manifold with negative sectional curvature, $u: M \rightarrow N$ induces an injection on the fundamental group and $\alpha \in K$ has infinite order. Let $N_{\alpha}$ be as in Lemma 6.5. Then there is a finite-sheeted covering $N_{\alpha} \rightarrow N$ such that $u: M \rightarrow N$ is homotopic to a map which factors through the inclusion of the fiber $M \rightarrow N_{\alpha}$.

Proof. Both $N_{\alpha}$ and $N$ are $K(\pi, 1)$ 's, prime and irreducible. By 9.11 of [13], $\pi_{1}\left(N_{\alpha}\right) \simeq \hat{K} \subset \pi_{1}(N)$ is a group of finite order in $\pi_{1}(N)$. It follows that $N_{\alpha}$ and the covering of $N$ corresponding to $\hat{K}$ are compact, prime, irreducible 3-manifolds with the same fundamental group. Therefore, they are diffeomorphic.

\section{REFERENCES}

1. W. Abikoff, Degenerating families of Riemann surfaces, Ann. of Math. (2) 105 (1977), 29-44.

2. ___ On boundaries of Teichmüller spaces and on Kleinian groups. III, Acta Math. 134 (1975), $211-237$

3. Illinois at Urbana-Champaign.

4. L. Bers, Uniformization, moduli and Kleinian groups, Bull. London Math. Soc. 4 (1972), 257-300.

5. $570-600$. , On boundaries of Teichmüller spaces and on Kleinian groups. I. Ann. of Math. (2) 91 (1970),

6. Spaces of degenerating Riemann surfaces, Discontinuous Groups and Riemann Surfaces, Ann. of Math. Studies, no. 79, Princeton Univ. Press, Princeton, N. J., 1974, pp. 43-55.

7. An extremal problem for quasiconformal mappings and a theorem of Thurston, Acta Math. 141 (1978), 73-98.

8. J. Eells and J. H. Sampson, Harmonic mappings of Riemannian manifolds, Amer. J. Math. 86 (1964), $109-159$.

9. R. Gulliver, Branched immersions of surfaces and reduction of topological type. II, Math. Ann. 230 (1977), 25-48.

10. R. Gulliver, R. Osserman and H. Royden, A theory of branched immersions of surfaces, Amer. J. Math. 95 (1973), 750-812.

11. P. Hartman, On homotopic harmonic maps, Canad. J. Math. 19 (1967), 673-687.

12. P. Hartman and A. Wintner, On the local behaviour of solutions of non-parabolic partial differential equations, Amer. J. Math. 75 (1953), 449-476.

13. J. Hempel, Three-manifolds, Ann. of Math. Studies, no. 86, Princeton Univ. Press, Princeton, N. J., 1976.

14. T. Jørgensen, Compact 3-manifolds of constant negative curvature fihering over the circle, Ann. of Math. (2) 106 (1977), 61-72.

15. L. Lemaire, Applications harmoniques de surfaces riemanniennes, J. Differential (jeom. 13 (1978), $51-78$.

16. L. A. Ljusternik, The topology of the calculus of variations in the large, Transl. Math. Mono., vol. 16, Amer. Math. Soc., Providence, R. I., 1966.

17. R. Schoen and S. T. Yau, The existence of incompressible minimal surfaces and the topology of three dimensional manifolds with non-negative scalar curvature, Ann. of Math. (2) 110 (1979), 127-142.

18. J. Sacks and K. Uhlenbeck, The existence of minimal immersions of 2-spheres, Ann. of Math. (2) 113 (1981), 1-24. 
19. D. Sullivan, Travaux de Thurston sur les groupes quasi-Fuchsiens et les variétés hyperboliques de dimension 3 fibrées sur $S^{1}$, Séminaire Bourbaki, 32e année (1979/80), no. 554, pp. 1-17.

20. W. Thurston, The geometry and topology of 3-manifolds, mimeographed notes, Princeton Univ., Princeton, N. J.

21. K. Uhlenbeck, Morse theory by perturbation with applications to harmonic maps, Trans. Amer. Math. Soc. 267 (1981), 569-583.

Department of Mathematics, Massachusetts institute of Technology, Cambridge, MasSACHUSETTS 02139

Department of Mathematics, University of Illinois at Chicago Circle, Chicago, Illinois 60680 